\title{
Evaluación de un Antioxidante Natural extraído del Marañón (Anacardium occidentale L.) para mejorar la Estabilidad Oxidativa del Biodiesel de Jatropha
}

\author{
Lorena Sánchez ${ }^{(1)}$, Jacqueline Chávez ${ }^{(1)}$, Luís A. Ríos ${ }^{(2)}$, Sandra M. Cardona ${ }^{(2)}$ * \\ (1) Grupo de Investigación en Producción y Transformación Agroindustrial (GIPTA), Universidad Popular \\ del Cesar, Carrera 40 Vía al Mar, Aguachica-Colombia (e-mail: loreny1986@gmail.com) \\ (2) Grupo Proceso Químicos Industriales, Facultad de ingeniería, Universidad de Antioquia UdeA, \\ Calle 70 No.52-21, Medellín, Colombia (e-mail: lariospfa@gmail.com; sacavi63@gmail.com)
}

${ }^{*}$ Autor a quien debe ser dirigida la correspondencia

Recibido Mar. 23, 2015; Aceptado May. 28, 2015; Versión final Jul. 28, 2015, Publicado Dic. 2015

\begin{abstract}
Resumen
Se evaluó un antioxidante natural extraído de la cáscara de la nuez de marañón (CNSL) para mejorar la estabilidad oxidativa del biodiesel de jatropha. Mediante el uso del equipo Rancimat Metrohm 873, se evaluó la estabilidad oxidativa a diferentes temperaturas, del biodiesel de jatropha puro y con adición de diferentes concentraciones de CNSL y de Propil Galato. Se encontró que a medida que aumenta la concentración de antioxidante, hay un incremento gradual en el tiempo de inducción, y a mayor temperatura de evaluación ocurre una disminución de esta variable. Se realizó el análisis estadístico de los resultados mediante tablas ANOVA y se describió la cinética de oxidación por la ley de velocidad de primer orden, se ajustó la velocidad de reacción de consumo de CNSL en el biodiesel de jatropha con la ecuación de Arrhenius y se obtuvo una energía de activación de $103.94 \mathrm{KJ} / \mathrm{mol}$.
\end{abstract}

Palabras clave: biodiesel de jatropha; Anacardium occidentale L.; cascara de nuez de Marañón; antioxidantes; cinética

\section{Evaluation of a Natural Antioxidant extracted from Cashew (Anacardium occidentale L.) to improve the Oxidative Stability of Jatropha Biodiesel}

\begin{abstract}
A natural antioxidant extracted from the shell of the cashew nut (CNSL) to improve the oxidative stability of jatropha biodiesel was evaluated. The oxidation stability at different temperatures of pure jatropha biodiesel and biodiesel with different concentrations of CNSL and propyl gallate was evaluated using the Metrohm 873 Rancimat equipment. It was found that there is a gradual increase in the induction time with the amount of antioxidant, and evaluation at higher temperature cause a decrease in this variable. Statistical analysis of the results was performed using ANOVA tables and oxidation can be described using a first order kinetics. The rate of consumption of CNSL in jatropha biodiesel was adjusted using the Arrhenius equation and activation energy of $103.94 \mathrm{~kJ} / \mathrm{mol}$ was obtained
\end{abstract}

Keywords: jatropha biodiesel; Anacardium occidentale L.; Cashew nut sell; antioxidants; kinetics 


\section{INTRODUCCIÓN}

En los últimos años, el aumento de las preocupaciones ambientales y de abastecimiento asociados a la utilización de combustibles fósiles por el agotamiento de las reservas de petróleo han generado a nivel mundial la búsqueda de nuevas fuentes de energía alternativa. En este contexto el biodiésel aparece como un combustible renovable para motores diésel, el cual se produce a partir de diferentes aceites vegetales y grasas animales tales como colza, soya, palma, jatropha, sebo de vaca y manteca de cerdo. La producción de biodiésel a partir de aceite de jatropha es una alternativa prometedora en la situación actual debido a su alto contenido de aceite y a su naturaleza no comestible (su uso no compite con la producción alimentaria), además, la jatropha puede cultivarse en tierras estériles y baldías, en condiciones simples, lo que se traduce en una materia prima de bajo costo (Sarin et al., 2010; Basir et al., 2015; Sánchez et al., 2015).

El biodiésel es amigable con el ambiente, su uso disminuye las emisiones contaminantes y la dependencia de los combustibles fósiles y, en términos de calidad, es similar al petro-diésel (Jain y Sharma, 2011). El biodiésel, sin embargo, presenta algunos inconvenientes a nivel de su estructura química, dada la naturaleza de los metilésteres que lo conforman, los cuales son fácilmente oxidables, debido a factores como la presencia de oxígeno, la exposición a la luz, las altas temperaturas y el contacto con los metales presentes en los tanques de almacenamiento o en las unidades de producción del mismo. La oxidación es una de las principales dificultades en la producción y comercialización del biodiésel y es motivo de preocupación en el sector automotriz ya que afecta el desempeño del motor (Zuleta et al., 2012a).

Para la evaluación de la oxidación del biodiésel existen reportados muchos métodos: índice de yodo, índice de peróxido, número acido, contenido de metilésteres, espectroscopía infrarroja, índice de estabilidad oxidativa (Sarin et al., 2010; Zuleta et al., 2012b), entre otros, los cuales son útiles para evaluar su susceptibilidad a la oxidación o determinar principalmente el grado de oxidación en que se encuentra un biocombustible. De estos métodos, el índice de estabilidad oxidativa es uno de los más importantes y se define como el punto de máximo cambio de la velocidad de oxidación, atribuido al incremento de la conductividad causado por la disociación de los ácidos carboxílicos volátiles generados durante el proceso de oxidación. Según la norma europea UNE-EN 14214 el biodiésel debe alcanzar una estabilidad oxidativa en un mínimo de 8 horas, medida por el método Rancimat a $110^{\circ} \mathrm{C}$ (UNE-EN 14214:2013).

Para mejorar significativamente la estabilidad oxidativa del biodiésel surge como alternativa la adición de antioxidantes ya sean naturales (tocoferoles) o sintéticos (propil galato, pyrogalol, tert-butil hidroquinona, terc-butil hidroxi anisol, tert-butil metil-fenol), los cuales inhiben el proceso de oxidación (Jain y Sharma, 2011; Xin et al., 2009). Los antioxidantes naturales presentes en frutas y vegetales, han venido siendo objeto de gran interés en los últimos años. Numerosos estudios han reportado que los compuestos fenólicos, presentes tanto en antioxidantes naturales como sintéticos, tienen una excelente actividad antioxidante al actuar como agentes reductores y captadores de radicales libres (Andrade et al., 2011).

El Marañón (Anacardium occidentale L.) es un árbol silvestre originario de las zonas tropicales de América, el cual pertenece a la familia Anacardiaceae. Es un importante cultivo comercial a nivel mundial, siendo la India el mayor productor, procesador y exportador de almendra de Marañón en el mundo (Andrade et al., 2011; Lafont et al., 2011; Carvalho et al., 2011; Kasiraman et al., 2012). En Colombia, esta especie se encuentra principalmente en la Costa Atlántica, en el valle del río Magdalena, en el valle del río Cauca, en los Llanos Orientales, Cundinamarca, la Amazonia y en las islas de San Andrés y Providencia (Corporación Autónoma Regional de Cundinamarca, 2004).

El Cashew Nut Shell Liquid (CNSL), conocido también como el líquido de la cascara de la nuez de Marañón (LCNM), es un recurso natural renovable, económico, de color oscuro, cáustico y viscoso, obtenido como subproducto durante el proceso de producción de almendras de la nuez de Marañón (Cheriyan y Abraham, 2010; Rodrigues et al., 2011). El CNSL es una fuente natural única para la producción de fenoles saturados e insaturados de cadena larga (ácido anacárdico, cardol, cardanol y 2-Metilcardol) y representa una buena alternativa como sustituto de los fenoles derivados petroquímicamente (Andrade et al., 2011; Maia et al., 2012). Ha sido estudiado como antioxidante para la estabilización de la gasolina, por poseer una estructura química similar a una de las sustancias que se utilizan como antioxidantes en los combustibles (Carvalho et al., 2011).

Dado el potencial de Colombia para la producción de Marañón en sus zonas cálidas y las políticas nacionales que promueven la producción de biodiésel de aceites naturales, esta investigación reviste gran importancia, porque busca evaluar un antioxidante natural extraído del Marañón, (CNSL) para mejorar la estabilidad oxidativa del biodiésel de jatropha. Existen varios trabajos relacionados con la estabilidad oxidativa de los biocombustibles (Sarin et al., 2010; Chen et al., 2011; Jain y Sharma., 2011; Zuleta et al., 2012b; Aguilar et al., 2015), pero muy pocos acerca de la evaluación del CNSL como antioxidante natural en 
biodiésel (Araújo et al., 2010.) y ninguno sobre la evaluación del CNSL como antioxidante en el biodiésel de jatropha. Desde este punto de vista, este trabajo pretende aportar nuevos conocimientos que pueden ser útiles como base para futuras investigaciones en este campo y que permitan promover la producción y comercialización del CNSL.

\section{MATERIALES Y MÉTODOS}

\section{Extracción y caracterización del antioxidante natural de la cascara de la nuez del marañón}

La nuez de marañón se recolectó de árboles de Anacardium occidentale L. ubicados en el municipio de Tamalameque, departamento del Cesar-Colombia. El antioxidante natural Cashew Nut Shell Liquid (CNSL), se extrajo a partir de las cascaras de la nuez del marañón que se obtuvieron después de la separación de las almendras.

Para la obtención del CNSL se siguió la metodología propuesta por Chuayjuljit et al. (2007), con una modificación en el tiempo de extracción, empleando para este caso 90 horas y agitación magnética. Este método consistió en una extracción simple a temperatura ambiente de la ciudad de Medellín-Antioquia (Promedio $24,2^{\circ} \mathrm{C}$ ), utilizando como solvente hexano p.a.: se mezclaron las cascaras reducidas de tamaño con el hexano usando la relación propuesta por Gaitán et al. (2003), (500ml solvente/100g cascaras). Durante el transcurso de las 90 horas se realizaron 6 rota-evaporaciones de la mezcla solvente - CNSL, una cada 15 horas para obtener el antioxidante natural y recuperar el solvente, que vuelve nuevamente al proceso de extracción. La separación se hizo en un rotavapor BUCHI, a una temperatura de $60{ }^{\circ} \mathrm{C} \mathrm{y} 75 \mathrm{rpm}$. El CNSL obtenido se caracterizó mediante índice de acidez y espectroscopía infrarroja por transformada de Fourier - Reflectancia total atenuada (FTIR-ATR), utilizando un espectrómetro IR Prestige 21, con accesorio de reflectancia total atenuada. Los espectros se obtuvieron en el rango de $4.000-500 \mathrm{~cm}^{-1}$.

\section{Producción y caracterización del biodiésel de jatropha}

Inicialmente se determinó el índice de acidez del aceite crudo de semilla de jatropha, para luego realizar una esterificación ácida para disminuir la acidez de este aceite. Las condiciones de reacción fueron: relación molar metanol/aceite de $3 / 1,1 \%$ de $\mathrm{H}_{2} \mathrm{SO}_{4}$ con respecto al aceite, temperatura de $60{ }^{\circ} \mathrm{C}$ y tiempo de reacción de 2 horas. El producto obtenido se dejó decantando 1 hora y se separó el aceite esterificado, el cual se utilizó para la producción del biodiésel.

El biodiésel de jatropha, se obtuvo por el método de transesterificación, usando una relación molar metanol/ aceite $9 / 1$ y KOH como catalizador $\left(0,7 \%\right.$ p/p en base al aceite), temperatura de $60{ }^{\circ} \mathrm{C}$ y tiempo de reacción de 1 hora. El producto obtenido se llevó a un embudo de decantación para separar el biodiésel crudo y la glicerina; el biodiésel se lavó con agua caliente hasta obtener un pH neutro en las aguas de lavado. Finalmente, al metiléster se le retiró la humedad residual, haciéndolo pasar a través de un lecho filtrante de sulfato de manganeso anhidro $\left(\mathrm{MnSO}_{4}\right)$, mediante el uso de un sistema de filtración al vacío. El biodiésel producido se caracterizó mediante índice de acidez y contenido de metiléster; este último se determinó mediante cromatografía gaseosa siguiendo la norma EN 14103 (UNE-EN 14103:2011) para lo que se utilizó un equipo Agilent 7890A con una columna capilar Agilent J\&W HP-Innowax, un detector de ionización de llama (FID), y tetradecano $\left(\mathrm{C}_{14} \mathrm{H}_{30}\right)$ como estándar interno. Se prepararon por duplicado muestras de biodiésel puro y aditivadas a diferentes concentraciones del antioxidante natural extraído (2.000; 4.000; 6.000 y 8.000 ppm de CNSL). La determinación del índice de acidez del CNSL, del aceite de jatropha y del biodiésel de jatropha se realizó según la norma NTC 218 (NTC 218, 1999), mediante titulación potenciométrica en un titulador automático 848 Titrino Plus Metrohm®.

\section{Evaluación de la estabilidad oxidativa}

Las estabilidades oxidativas del biodiésel de jatropha puro y aditivado a diferentes concentraciones de CNSL (2.000, 4.000, 6.000 y 8.000 ppm) se evaluaron por duplicado a diferentes temperaturas (90, 100 y $110^{\circ} \mathrm{C}$ ). También se evaluó la estabilidad oxidativa del biodiésel de jatropha a temperatura de $110^{\circ} \mathrm{C}$, aditivado con 2.000 y 8.000 ppm de Propil Galato (PG, antioxidante sintético), con el fin de determinar el efecto de los factores temperatura, concentración y clase de antioxidante en la variable dependiente tiempo de inducción (Ti). La evaluación se hizo en un equipo 873 Biodiésel Rancimat (Metrohm®), siguiendo el protocolo establecido en la norma EN 14112 (UNE-EN 14112:2003). Los resultados obtenidos de los tiempos de inducción de las diferentes muestras, se analizaron mediante un tratamiento matemático estadístico, utilizando el programa Statgraphics, a través del cual se obtuvo la ANOVA multifactorial, procedimiento que estableció un análisis de varianza, para determinar qué factores tienen un efecto estadísticamente significativo sobre tiempo de inducción. También se evaluó la significancia de las interacciones entre ellos. 


\section{Cinética de la oxidación del biodiésel estabilizado con CNSL}

Empleando los resultados obtenidos en la etapa anterior de la evaluación de la estabilidad oxidativa a diferentes concentraciones de CNSL a tres temperaturas, se describe la cinética de oxidación del biodiésel de jatropha estabilizado con CNSL por la ley de velocidad de primer orden y se ajusta la velocidad de reacción de consumo de CNSL con la ecuación de Arrhenius para determinar la energía de activación la cual da cuenta de la sensibilidad del antioxidante a la temperatura (Xin et al., 2009).

\section{RESULTADOS Y DISCUSIÓN}

\section{Extracción y caracterización del antioxidante natural de la cascara de la nuez del marañón}

Del proceso de extracción se obtuvo un líquido viscoso, de color marrón rojizo, que constituyó aproximadamente un $23,5 \%$ en peso de las cáscaras de la nuez empleadas, valor que al ser comparado con otras investigaciones, se encuentra por encima del reportado para especies de marañón cultivadas en África (15-20\%) y por debajo de especies de la India (25-30 \%) (Kasiraman et al., 2012), de Brasil (32\%) y de la variedad criollo Llanero de Colombia (38,2\%) (Gaitan et al., 2003). Este rendimiento depende en gran parte de la variedad, del método de extracción y del tipo de solvente utilizado.

El índice de acidez del antioxidante natural extraído (CNSL) fue de $91,94 \mathrm{mg} \mathrm{KOH} / \mathrm{g} \mathrm{CNSL}$, el cual se atribuye a la presencia del ácido anacárdico en este líquido, el cual representa entre el 46-70\%, en comparación con los otros componentes como cardol (15-31\%), Cardanol (5-22 \%) y 2-Metilcardol (trazas) (Cheriyan y Abraham, 2010; Rodrigues et al., 2011; Maia et al., 2012). El espectro FTIR del CNSL se presenta en la figura 1, en la cual se observa la presencia de varios grupos funcionales característicos de esta molécula. La absorción de los fenoles se asocia con la vibración de los enlaces O-H y C-O; en este espectro la vibración de estiramiento del enlace $\mathrm{O}-\mathrm{H}$ se aprecia alrededor de los $3.400 \mathrm{~cm}^{-1}$, es una absorción intensa y amplia desde 3.600 hasta $3.200 \mathrm{~cm}^{-1}$ aproximadamente; la vibración del enlace C-O aparece entre $1260-1000 \mathrm{~cm}^{-1}$. También se observa el estiramiento asimétrico y simétrico de los grupos metilos y metileno en el rango de frecuencias comprendido entre $3000-2840 \mathrm{~cm}^{-1}$. El estiramiento asimétrico del enlace $\mathrm{C}=\mathrm{O}$ se manifiesta entre 1725 a $1700 \mathrm{~cm}^{-1}$, caracterizado por una banda intensa típica de este grupo funcional. El estiramiento del grupo $\mathrm{O}-\mathrm{H}$ del ácido carboxílico es evidente en el rango de frecuencias entre $2400-3600 \mathrm{~cm}^{-1}$. Las vibraciones de estiramiento del enlace $\mathrm{C}=\mathrm{C}$ propias de bencenos pueden apreciarse en el rango de frecuencias que va desde 1670 a $1640 \mathrm{~cm}^{-1}$. El espectro obtenido fue similar al reportado por Chuayjuljit et al. (2007) en su investigación.

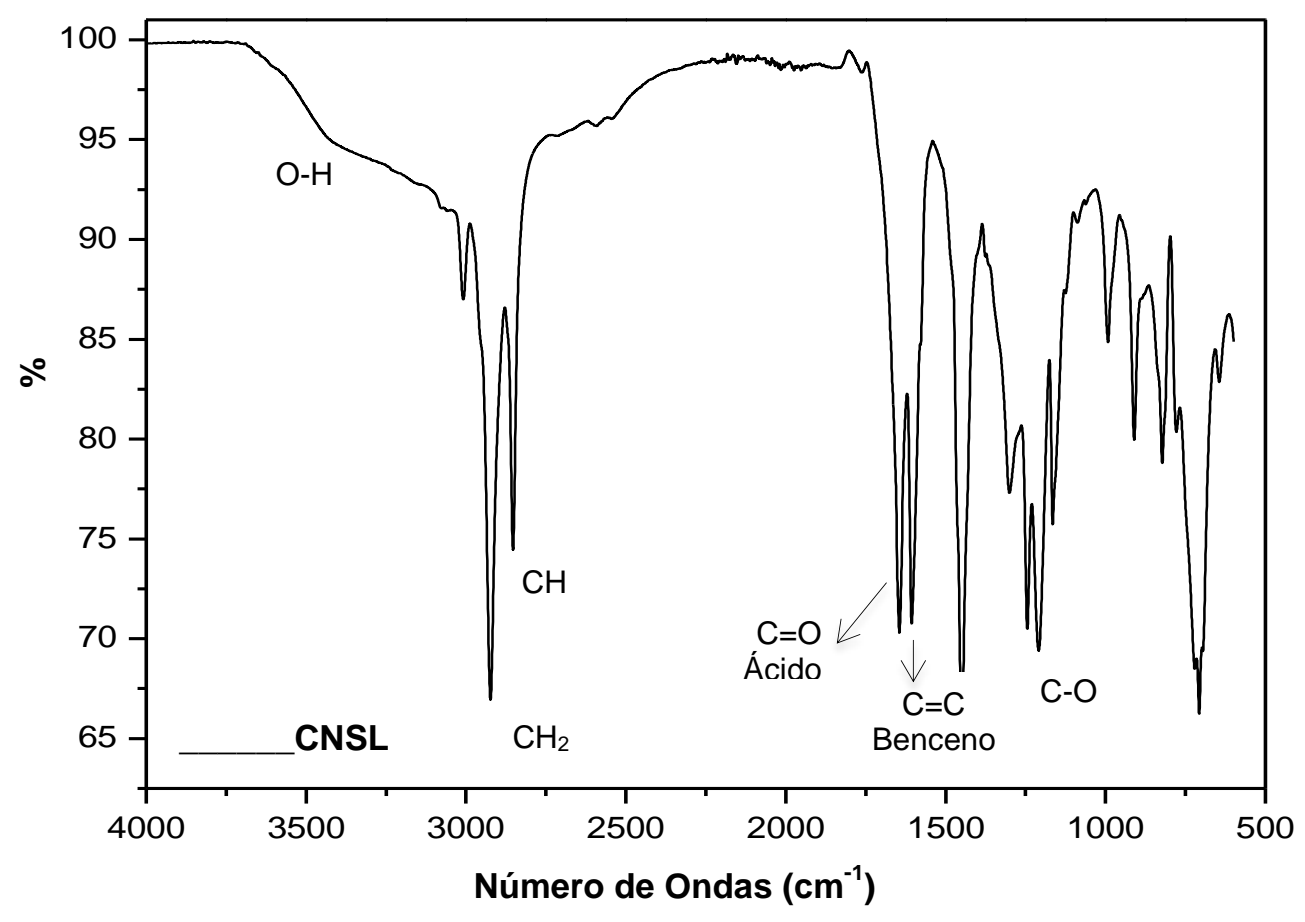

Fig. 1: Espectro FTIR del CNSL. 


\section{Producción y caracterización del biodiésel de jatropha}

El índice de acidez del aceite crudo de semilla de jatropha fue de $17,329 \mathrm{mg} \mathrm{KOH} / \mathrm{g}$ aceite, valor relativamente alto, por lo que fue necesario realizar una esterificación ácida con el fin de disminuir este valor. El índice de acidez logrando finalmente fue de $0,276 \mathrm{mg} \mathrm{KOH} / \mathrm{g}$ muestra, valor óptimo para ser transesterificado. El índice de acidez del biodiésel de aceite de jatropha producido fue de $0,305 \mathrm{mg} \mathrm{KOH} / \mathrm{g}$ muestra y el contenido de metiléster fue de $98,2 \%$, lo que indica que hubo una alta conversión de los ácidos grasos en la reacción de transesterificación. Estos dos parámetros cumplen con los requisitos de calidad exigidos para el biodiésel a ser mezclado con el diésel petroquímico, según la Resolución 90963 (Resolución 90963, 2014), de los Ministerios de Minas y Energía, y de Ambiente y Desarrollo Sostenible (índice de acidez: máximo 0,5 mg KOH/g; y contenido de metiléster: mínimo 96,5\%). El perfil de ácidos grasos del biodiésel de jatropha determinado por cromatografía gaseosa se muestra en la Tabla 1, donde se observa que este biocombustible está compuesto principalmente por metilésteres de ácido oleico y linoleico, es decir, es altamente insaturado.

Tabla 1: Composición de metilésteres del biodiésel de jatropha

\begin{tabular}{|l|c|}
\hline Metiléster & Composición $(\% \mathrm{p} / \mathrm{p})$ \\
\hline Miristato & 0,059 \\
\hline Palmitato & 13,910 \\
\hline Palmitoleato & 0,730 \\
\hline Heptadecanoato & 0,147 \\
\hline Estearato & 7,360 \\
\hline Oleato & 44,720 \\
\hline Linoleato & 30,780 \\
\hline Linolenato & 0,157 \\
\hline Eicosanato & 0,246 \\
\hline Eicosenato & 0,108 \\
\hline Metilésteres saturados & 21,700 \\
\hline Metilésteres monoinsaturados & 45,550 \\
\hline Metilésteres poliinsaturados & 30,940 \\
\hline
\end{tabular}

Efecto de la temperatura y la concentración de antioxidante en la estabilidad oxidativa

Los resultados de la estabilidad oxidativa del biodiésel de jatropha puro y aditivado a diferentes concentraciones del antioxidante natural CNSL se presentan en la figura 2 (en la tabla 2 se presentan las ANOVA correspondiente). En la figura 2 puede observarse que a medida que aumenta la temperatura de evaluación del biodiésel se produce un menor tiempo de inducción, y que a medida que aumenta la concentración del antioxidante CNSL se produce un aumento en dicho tiempo de inducción. La norma Europea UNE-EN 14214 establece como mínimo un tiempo de inducción de $8 \mathrm{~h}$ para los biodiésel evaluados a una temperatura de $110^{\circ} \mathrm{C}$. El resultado obtenido para el biodiésel de jatropha sin aditivar $(6,07$ h) no cumple con lo establecido en esta norma. Si éste se compara con otros resultados obtenidos para otros tipos de biodiésel de jatropha (3,28 h y 3,95 h; Sarin et al., 2010; Rios et al., 2012), el resultado alcanzado en esta investigación lo supera con creces, aunque este hecho puede explicarse dada la alta variedad que existe en los métodos utilizados para la producción de biodiésel y en las características de las materias primas utilizadas. La razón primordial podría estar en el porcentaje de metilésteres monoinsaturados presentes en el biodiésel de jatropha, bastante superior al de poliinsaturados (tabla 1). En general la estabilidad oxidativa del biodiésel depende de la composición de los metilésteres de ácidos grasos y de la presencia de antioxidantes naturales en las materias primas (Chen et al., 2011).

El análisis de varianza para los tiempos de inducción del biodiésel de jatropha puro y aditivado se presenta en la tabla 2; en esta se puede observar la contribución de los factores Temperatura y Concentración sobre la variabilidad del tiempo de inducción. Puesto que se ha escogido la suma de cuadrados Tipo III (por omisión), la contribución de cada factor se midió eliminando los efectos de los demás factores. Los valores- 
$\mathrm{P}$ prueban la significancia estadística de cada uno de los factores. Como se puede observar los 3 valores-P son menores que 0,05 , lo que indica que estos factores tienen un efecto estadísticamente significativo sobre el Tiempo de Inducción, con un 95,0 \% de nivel de confianza y que si existe interacción entre ellos. Todas las razones- $\mathrm{F}$ se basan en el cuadrado medio del error residual.

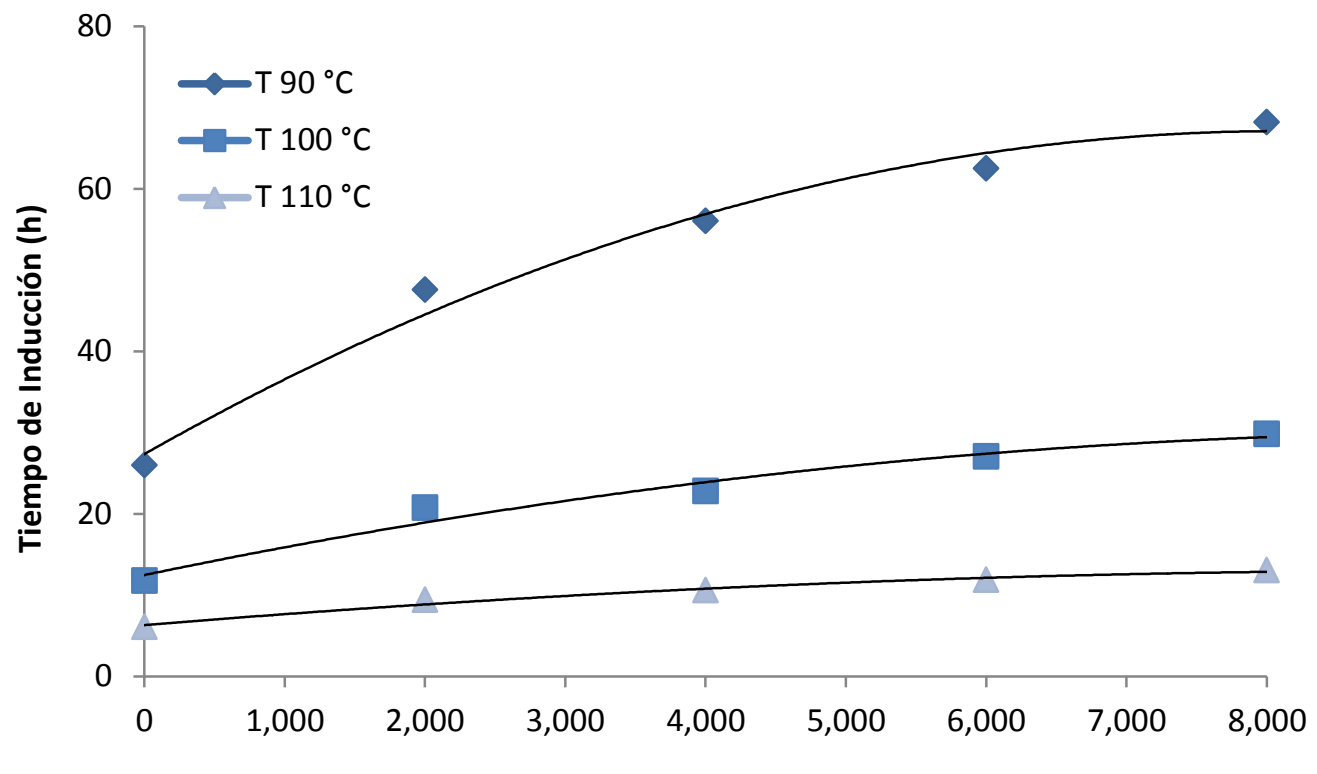

Concentraciones de CNSL (ppm)

Fig. 2: Influencia de la concentración de CNSL y la temperatura de evaluación, en la estabilidad oxidativa del biodiésel de jatropha.

Tabla 2: Análisis de varianza para Tiempo de Inducción

\begin{tabular}{|l|c|c|c|c|c|}
\hline Fuente & Suma de cuadrados & Gl & Cuadrado medio & Razón-F & Valor-P \\
\hline \multicolumn{7}{|c|}{ Efectos principales } \\
\hline A: Temperatura & 9481,671 & 2 & 4740,835 & 6205,650 & 0,000 \\
\hline B: Concentración CNSL & 1940,373 & 4 & 485,093 & 634,980 & 0,000 \\
\hline \multicolumn{7}{|c|}{ Interacciones } \\
\hline AB & 881,451 & 8 & 110,181 & 144,230 & 0,000 \\
\hline Residuos & 12,223 & 16 & 0,764 & & \\
\hline Total (Corregido) & 12356,300 & 30 & & & \\
\hline
\end{tabular}

En la figura 3 se muestra un comparativo de la influencia de un antioxidante sintético (Propil Galato, PG) y uno natural (CNSL) sobre la estabilidad oxidativa del biodiésel de jatropha evaluada a $110^{\circ} \mathrm{C}$; en ambos casos se observa un aumento gradual en el tiempo de inducción con un aumento de la concentración de antioxidante adicionado al biocombustible. EI CNSL fue menos eficiente que PG pero se debe considerar que en este estudio se utilizó el CNSL sin purificar, es decir, sin la separación de las sustancias antioxidantes presentes en su composición (ácido anacárdico, cardol y cardanol), buscando evaluar el líquido tal como sale del proceso de producción de la almendra, sin incurrir en los costos adicionales que implicaría su purificación. El menor efecto antioxidante del CNSL se puede explicar a partir de las estructuras moleculares: el CNSL está compuesto en mayor porcentaje por ácido anacárdico (46-70\%), el cual posee un grupo $\mathrm{OH}$ unido al anillo aromático, en comparación con el PG que presenta 3 grupos OH en su estructura, los cuales permiten la formación de complejos entre radicales libres y radicales antioxidantes logrando de esta manera la estabilización de los lípidos (Sarin et al., 2010).

En la tabla 3 se presentan la ANOVA correspondiente a los resultados anteriores, en la cual se observa que para un nivel de confianza del 95,0 \%, los 2 valores-P son menores que 0,05 , lo que indica que estos factores tienen un efecto estadísticamente significativo sobre el Tiempo Inducción, reportando mayor significancia el tipo de antioxidante utilizado, en comparación con la concentración. 


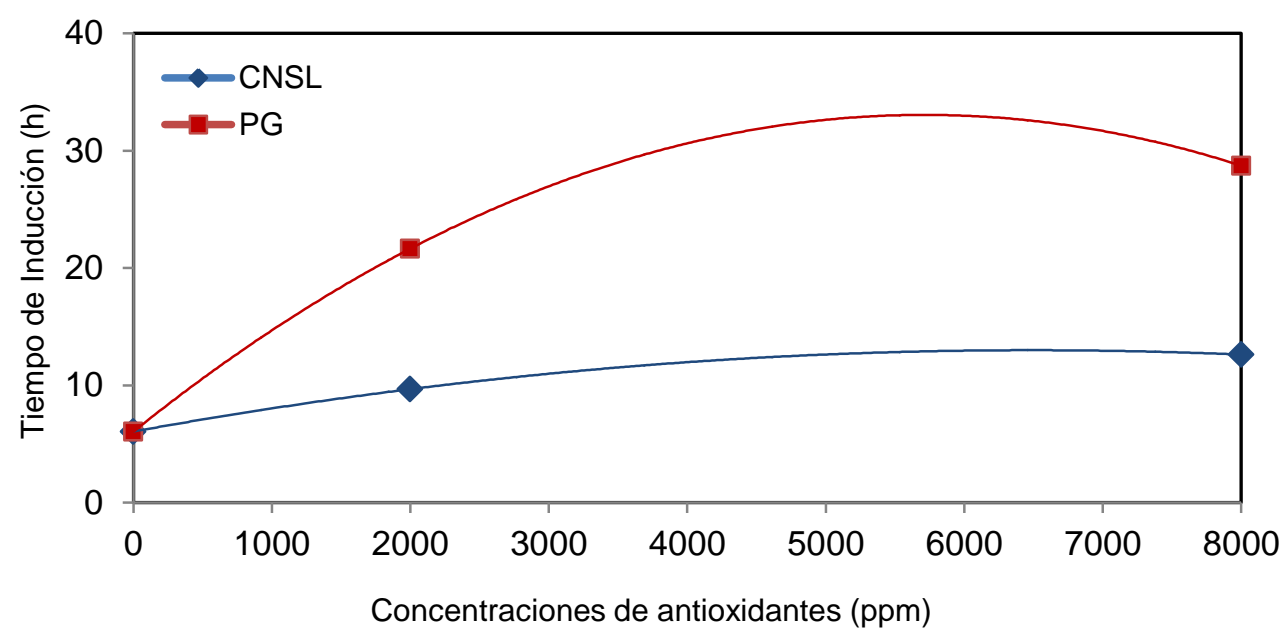

Fig. 3: Influencia del PG y el CNSL, en la estabilidad oxidativa del biodiésel de jatropha evaluada a $110^{\circ} \mathrm{C}$.

Tabla 3: Análisis de Varianza para Tiempo Inducción

\begin{tabular}{|l|c|c|c|c|c|}
\hline Fuente & Suma de cuadrados & Gl & Cuadrado medio & Razón-F & Valor-P \\
\hline \multicolumn{7}{|c|}{ Efectos principales } \\
\hline A: Antioxidante & 394,524 & 1 & 394,524 & 171,86 & 0,0000 \\
\hline B: Concentración & 50,200 & 1 & 50,200 & 21,87 & 0,0055 \\
\hline Residuos & 11,478 & 5 & 2,295 & & \\
\hline Total (Corregido) & 456,202 & 7 & & & \\
\hline
\end{tabular}

Cinética de la oxidación del biodiésel cuando es estabilizado con CNSL

La oxidación de compuestos orgánicos es un proceso de reacción en cadena bastante complejo que consta de varias etapas elementales: iniciación, propagación y terminación (Xin et al., 2009). La adición de antioxidantes retarda la ocurrencia de este fenómeno y, en este caso, el CNSL actúa como agente secuestrador de radicales libres en las etapas de iniciación y propagación del proceso oxidativo (Araujo et al., 2010). Como se mencionó en la metodología, la cinética de reacción se modelo asumiendo que la reacción es de primer orden con respecto a la concentración de CNSL (Xin et al. 2009). Las curvas de la figura 2 parecen ser no lineales, probablemente son función logaritmo exponencial; este mecanismo se puede describir por medio de una reacción cinética de primer orden. Los antioxidantes en el biodiésel tienen un papel importante en la determinación de la duración del periodo de inducción. Al inicio de la prueba Rancimat la concentración de CNSL es $\mathrm{C}_{0}$. Con el incremento del tiempo de oxidación, la concentración de CNSL disminuye y al final del periodo de inducción esta concentración se convierte en $\mathrm{C}_{\mathrm{cr}}$, valor de la concentración de CNSL por debajo de la cual el antioxidante no tiene efecto en retardar la oxidación del biodiésel. Por lo tanto, la velocidad de consumo de antioxidante es considerada como la base de la cinética de reacción de primer orden, para la cual, la ecuación de la velocidad es:

$\frac{d C}{d t}=-k \cdot C$

Donde $\mathrm{C}$ es la concentración de CNSL en cualquier tiempo, $\mathrm{C}_{0}$ y $\mathrm{C}_{\mathrm{cr}}$ son las concentraciones inicial y critica de CNSL, $t_{i}$ es el tiempo de inducción medido a través del método Rancimat y k es la constante de reacción del consumo de CNSL. Integrando la ecuación (1) en de los rangos de concentración de $\mathrm{C}_{0}$ a $\mathrm{C}_{\mathrm{cr}}$ y en los límites de tiempo de $t_{\mathrm{i} 0}$ a $t_{\mathrm{i}}$ resulta la ecuación (2):

$\ln \mathrm{C}_{0}-\ln \mathrm{C}_{\mathrm{cr}}=\mathrm{kt}_{\mathrm{i}}-\mathrm{kt} \mathrm{t}_{\mathrm{iO}}$

Donde $t_{i 0}$ es el tiempo de inducción medido por el método Rancimat para el biodiésel sin la adición del antioxidante natural. Como se mencionó antes, cuando la concentración de antioxidante es menor que $\mathrm{C}_{\mathrm{cr}}$, el tiempo de inducción es igual que sin la adición de antioxidante $t_{i 0}$. Por lo tanto, la ecuación (2) puede escribirse así: 
$\ln \mathrm{C}_{0}=\mathrm{k}\left(\mathrm{t}_{\mathrm{i}}-\mathrm{t}_{\mathrm{i} 0}\right)+\ln \mathrm{C}_{\mathrm{cr}}$

Esta ecuación muestra una relación lineal entre la concentración inicial de antioxidante $\mathrm{C}_{\circ}$ y el tiempo de inducción $\mathrm{t}_{\mathrm{i}}$. El término $\left(\mathrm{t}_{\mathrm{i}}-\mathrm{t}_{\mathrm{i}}\right)$ significa el cambio en el periodo de inducción después de la adición y sin la adición de antioxidante.

En la figura 4 se muestra la dependencia del tiempo de inducción del biodiésel de jatropha con el logaritmo natural (In) de la concentración de CNSL a temperatura de 90,100 y $110{ }^{\circ} \mathrm{C}$. Se muestran además las líneas de tendencia con su respectiva ecuación y el valor de $R$. Los datos se ajustan a líneas rectas con el fin de adoptar la ecuación de la velocidad de primer orden. Como se muestra en la tabla 4 las líneas muestran un buen grado de correlación ya que todos los coeficientes son mayores que 0,9.

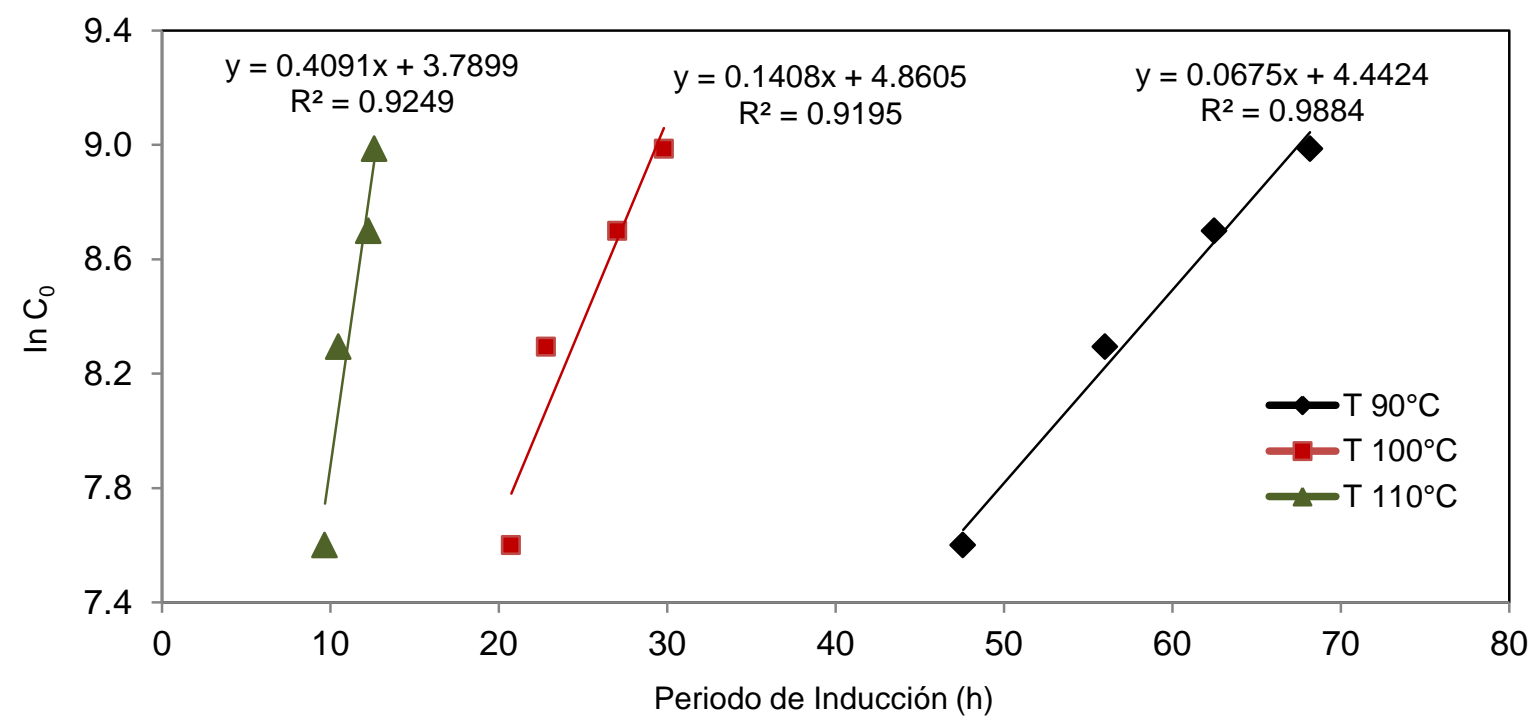

Fig. 4: Dependencia del tiempo de inducción del biodiésel de jatropha con el logaritmo natural (In) de la concentración de CNSL a temperatura de 90,100 y $110^{\circ} \mathrm{C}$.

La concentración crítica $\mathrm{C}_{\mathrm{cr}}$ de antioxidante puede determinarse por extrapolación de la línea recta del periodo de inducción sin la adición del mismo (Xin et al., 2009). La constante de reacción k que se muestra en la tabla 4 a varias temperaturas es igual a la pendiente de la línea recta. Como se puede observar $k$ incrementa con la temperatura siendo mayor a $110^{\circ} \mathrm{C}$. Altas constantes de reacción a altas temperaturas conducen a una velocidad más rápida de consumo de antioxidante y periodos de inducción más cortos.

Tabla 4: Constante ( $k$ ) para el consumo de CNSL a varias temperaturas y el coeficiente de correlación (R2) calculado asumiendo la ley de la velocidad de primer orden

\begin{tabular}{|c|c|c|c|}
\hline Temperatura $\left({ }^{\circ} \mathrm{C}\right)$ & $\mathrm{k}, \mathrm{h}-1$ & $\mathrm{Ccr}(\mathrm{ppm})$ & $\mathrm{R} 2$ \\
\hline 90 & 0,0675 & 85 & 0,9884 \\
\hline 100 & 0,1408 & 129 & 0,9195 \\
\hline 110 & 0,4091 & 44 & 0,9249 \\
\hline
\end{tabular}

Para comprobar si los valores de k calculados satisfacen la ecuación de Arrhenius, expresión matemática que se utiliza para comprobar la dependencia de la constante de velocidad o cinética de una reacción química con respecto a la temperatura a la que se lleva a cabo esa reacción, se realizó el gráfico de In $\mathrm{k}$ versus $\mathrm{T}^{-1}$ (figura 5), para el CNSL basado en la siguiente ecuación:

$\ln k=-\frac{E_{a}}{R T}+B$

$k=A \exp \left(-\frac{E_{a}}{R T}\right)$ 
Donde $E_{a}$ es la energía de activación, $R$ es la constante de la ley del gas ideal $(8.314 \mathrm{~J} / \mathrm{mol} \mathrm{K})$, B es la constante que intercepta la línea recta con el eje vertical y $\mathrm{A}$ es el factor pre-exponencial.

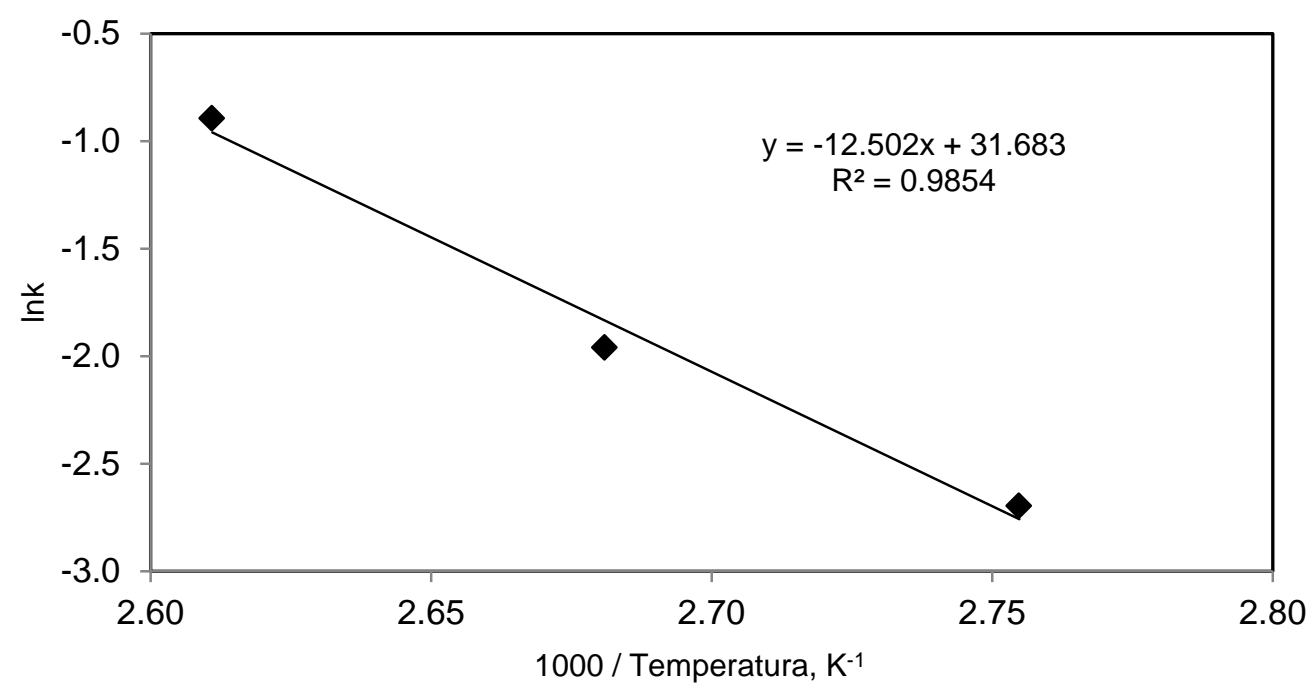

Fig. 5: Dependencia de la temperatura de la constante de velocidad del consumo de CNSL.

Los datos obtenidos para el experimento realizado están de acuerdo con la ley de Arrhenius. La energía de activación indica el grado de sensibilidad del antioxidante a la temperatura, de forma que a mayor valor de la energía de activación mayor sensibilidad a la temperatura y viceversa. La energía de activación calculada para el consumo de CNSL fue $103,94 \mathrm{KJ} / \mathrm{mol}$, valor que se considera bajo si se compara con los valores típicos de la energía de activación para la mayoría de reacciones (entre 40 a $400 \mathrm{~kJ} / \mathrm{mol}$ ) (Xin et al., 2009).

\section{Tiempo de inducción obtenido por el método de extrapolación}

Buscando obtener una relación que permita calcular el tiempo de inducción a temperaturas de las condiciones reales de almacenamiento, en la figura 6 se representa el logaritmo del tiempo de inducción en función de la temperatura del biodiésel de jatropha estabilizado con diferentes concentraciones de CNSL. En la misma puede observarse que el periodo de inducción y la temperatura tienen una alta correlación lineal. Se ha reportado que existe una relación lineal entre el periodo de inducción y la temperatura de los triglicéridos sin la adición de antioxidante (Xin et al., 2009). Si el mecanismo de consumo del antioxidante en el biodiésel no cambia a temperatura ambiente, las líneas rectas en la figura 6 pueden ser extrapoladas a baja temperatura para obtener el tiempo de inducción a temperaturas reales de almacenamiento (Xin et al., 2009). La figura 7 muestra el resultado del tiempo de inducción obtenido por el método de extrapolación de los datos de la figura 6 , donde se observa que para garantizar un año de almacenamiento del biodiésel de jatropha, a una temperatura de $25^{\circ} \mathrm{C}$, sin que sufra degradación alguna, se requiere la adición de 2.009 ppm de CNSL.

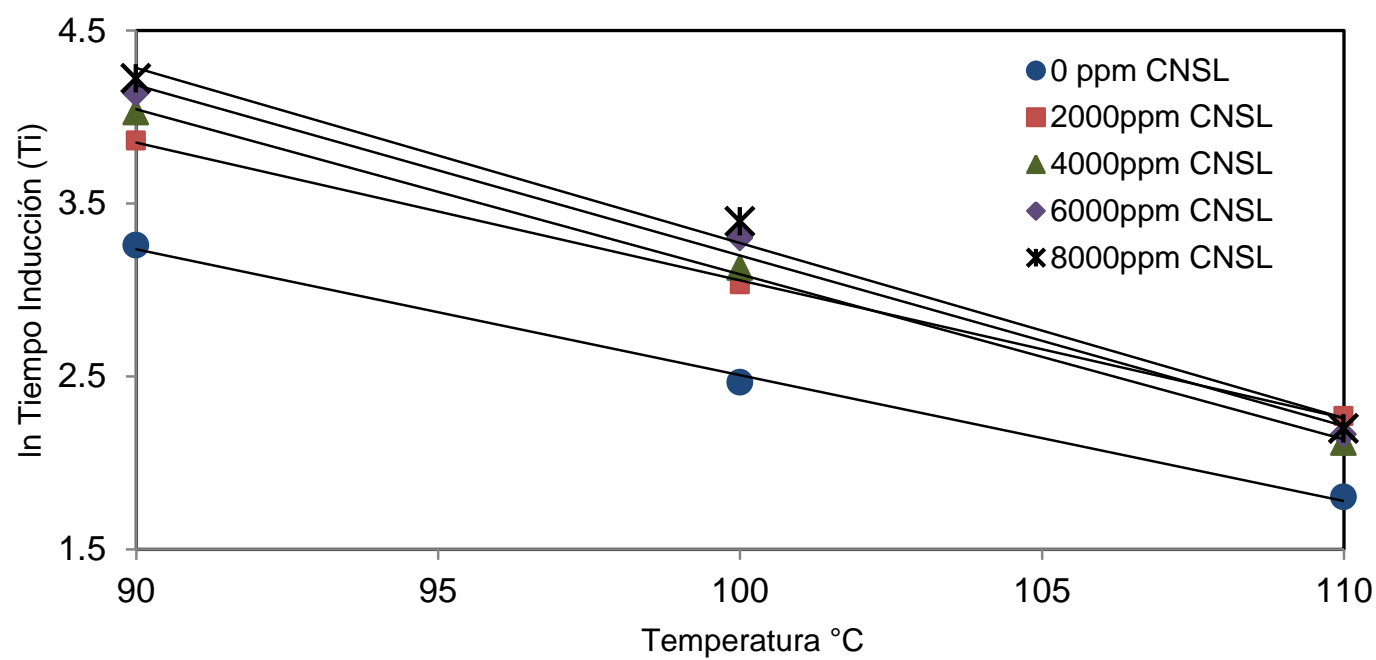

Fig. 6: Logaritmo del tiempo de inducción en función de la temperatura para el biodiésel de jatropha estabilizado con varias concentraciones de CNSL. 


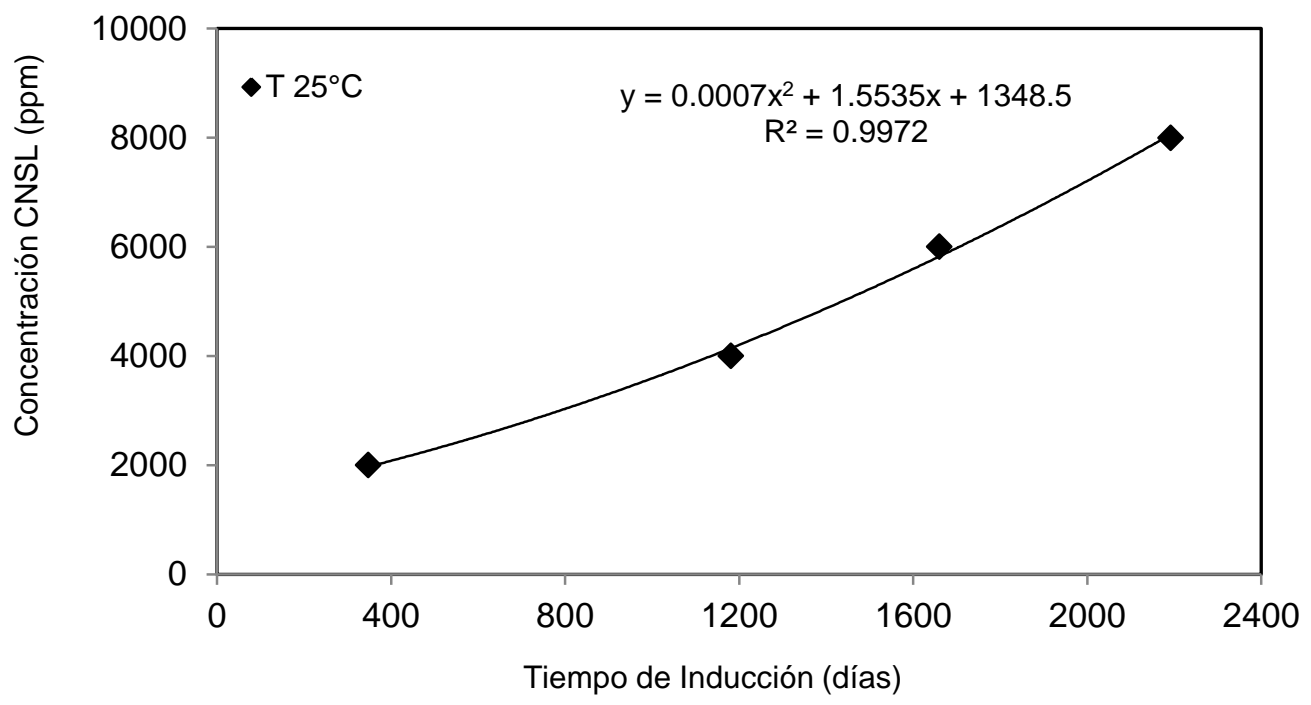

Fig. 7: Tiempo de inducción del biodiésel a temperatura ambiente de $25^{\circ} \mathrm{C}$ estabilizado con varias concentraciones de CNSL obtenido por el método de extrapolación.

Durante la etapa inicial en la oxidación de grasas y derivados lípidos las reacciones en cadena son suprimidas por agentes que rompen las cadenas por ejemplo los antioxidantes. Esta etapa es definida como el periodo de inducción. Seguida de esta etapa reacciones de oxidación proceden a la descomposición de productos primarios de oxidación (hidroperóxidos) produciendo una mezcla de productos secundarios incluyendo ácidos carboxílicos de cadena corta, cetonas, aldehídos, mono y dihidroxi compuestos y también polímeros o sedimentos. Como resultado después del periodo de inducción no solo se degradan rápidamente las propiedades principales del biocombustible, sino que también más adelante ocasionan problemas de funcionamiento del motor. Como la duración del tiempo de inducción es un factor importante en la vida comercial del biodiésel, largos periodos de inducción en la prueba de Rancimat presentan un mayor tiempo de almacenamiento tolerable para este biocombustible (Xin et al., 2009).

\section{CONCLUSIONES}

La producción de antioxidante extraído de la nuez de Marañón, podría ser económicamente viable en el país, ya que Colombia cuenta con las condiciones agroecológicas necesarias para su producción Sin embargo, es necesario profundizar en las investigaciones sobre este antioxidante.

El líquido extraído del Marañón (CNSL) logró mejorar la estabilidad oxidativa del biodiésel de jatropha, aun cuando este líquido se usó sin purificarse. Se esperaría que si el líquido se concentrara en las especies con mayor cantidad de grupos hidroxilo (cardol y 2-metilcardol) se mejoraría su poder antioxidante. Se confirmó que este líquido posee propiedades antioxidantes convirtiéndose en una alternativa potencial como sustituto de antioxidantes derivados de la industria petroquímica.

El proceso de oxidación del biodiésel de jatropha puede ser descrito con una alta precisión por una cinética de primer orden, cuando se evaluó en un rango de concentración del antioxidante CNSL entre 2.000 y 8.000 ppm y temperatura entre 90 a $110^{\circ} \mathrm{C}$. El bajo valor de la energía de activación calculada para el consumo de CNSL indica que el consumo de CNSL en el biodiésel de jatropha tiene baja sensibilidad a la temperatura.

El método de extrapolación permite predecir el tiempo de estabilidad al almacenamiento a diferentes temperaturas y encontrar las concentraciones requeridas de antioxidante que pueden alargar la vida del biocombustible. En este estudio se encontró que el biodiésel de jatropha puede permanecer 6 meses en almacenamiento a una temperatura de $25^{\circ} \mathrm{C}$, con la adición de $1655 \mathrm{ppm}$ del antioxidante natural extraído del Marañón (CNSL); este tiempo es aproximadamente el período de rotación del biodiésel en almacenamiento durante su comercialización.

\section{AGRADECIMIENTOS}

Los autores de este trabajo agradecen a: El programa Joven Investigador de COLCIENCIAS, los grupos de investigación GIPTA de la Universidad Popular del Cesar Seccional Aguachica y PQI de la Universidad de Antioquia y al Ingeniero Agroindustrial, M.I. Ernesto Camilo Zuleta Suarez por su asesoría. 


\section{NOTACIÓN}

A: $\quad$ Factor pre-exponencial

B: Constante que intercepta la línea recta con el eje vertical

C: Concentración de CNSL

$\mathrm{C}_{\mathrm{cr}}$ : Concentración critica del antioxidante en el biodiésel

$\mathrm{C}_{0}$ : Concentración inicial del antioxidante en el biodiésel

$E_{a}$ : Energía de activación

k: $\quad$ Constante de reacción de consumo de CNSL

R: Constante de la ley del gas ideal $(8.314 \mathrm{~J} / \mathrm{mol} \mathrm{K})$

$\mathrm{T}$ : Temperatura de reacción

$\mathrm{t}_{\mathrm{i}}$ : $\quad$ Tiempo de inducción medido por el método Rancimat

$t_{i 0}$ : Tiempo en el cual se adiciona el antioxidante natural

\section{REFERENCIAS}

Aguilar, C.A., y otros tres autores, Evaluación de la Estabilidad Oxidativa del Biodiesel de Jatropha (Jatropha curcas L.) mediante el uso de Antioxidantes Sintéticos y Biodiesel de Palma, Información Tecnológica: 26(2), 51-60 (2015)

Andrade, T.d.J.A.d.S., y otros seis autores, Antioxidant properties and chemical composition of technical Cashew Nut Shell Liquid (tCNSL), Food Chemistry: 126(3), 1044-1048 (2011)

Araujo, F.D.d.S, Moura, C.V.R.d y Chaves, M.H., Biodiésel Metílico de Dipteryx lacunifera: preparação, caracterização e efeito de antioxidantes na estabilidade à oxidação, Química Nova: 33(8) 1671-1676 (2010)

Carvalho, A.L.N., y otros seis autores, Acute, Subacute Toxicity and Mutagenic Effects of Anacardic Acids from Cashew (Anacardium occidentale Linn.) in Mice, Journal of Ethnopharmacology: 135(3), 730-736 (2011)

Corporación Autónoma Regional de Cundinamarca, C., ed. Vegetación del Territorio CAR, 450 especies de sus Llanuras y Montañas, $1^{\underline{a}}$ ed., 393, Bogotá D.C., Colombia (2004)

Chen, Y.-H. y Luo, Y.-M., Oxidation Stability of Biodiesel Derived from Free Fatty Acids Associated with Kinetics of Antioxidants, Fuel Processing Technology: 92(7), 1387-1393 (2011)

Cheriyan, S. y Abraham, E.T., Enzymatic Bioremediation of Cashew Nut Shell Liquid Contamination, Journal of Hazardous Materials: 176(1-3), 1097-1100 (2010)

Chuayjuljit, S., Rattanametangkool, P. y Potiyaraj, P., Preparation of Cardanol-Formaldehyde Resins from Cashew Nut Shell Liquid for the Reinforcement of Natural Rubber, Journal of Applied Polymer Science: 104(3), 1997-2002 (2007)

Basir, A.F., Datta, S., Kumar P.R, Studies on biodiesel production from Jatropha Curcas oil using chemical and biochemical methods - A mathematical approach, Fuel: 158, 503-511 (2015)

Gaitán, S. y otros tres autores, Efecto de la Temperatura de Industrialización de la Nuez de Marañón en la Actividad Antibacteriana en Streptococcu smutans del Líquido de la Cáscara, Revista Colombiana de Química, 32(2), 103 - 112 (2003)

Jain, S. y Sharma, M.P., Oxidation Stability of Blends of Jatropha Biodiesel with Diesel, Fuel: 90(10), 3014$3020(2011)$

Kasiraman, G., Nagalingam, B. y Balakrishnan, M. Performance, Emission and Combustion Improvements in a Direct Injection Diesel Engine Using Cashew Nut Shell Oil as Fuel with Camphor Oil Blending, Energy: 47(1): 116-124 (2012)

Lafont, J., Paez, S. y Portacio, A., Extracción y Caracterización Fisicoquímica del Aceite de la Semilla (Almendra) del Marañón (Anacardium occidentale L), Información Tecnológica: 22(1), $51-58$ (2011) 
Maia, F.J.N. y otros ocho autores, Electrochemical and Computational Studies of Phenolic Antioxidants from Cashew Nut Shell Liquid, Electrochimica Acta: 79(0), 67-73 (2012)

NTC 218, Grasas y Aceites Vegetales y Animales, Determinación del Índice de Acidez y de la Acidez, 1-14, Colombia (2011)

Resolución 90963, Ministerios de Minas y Energía, y Ministerio de Ambiente y Desarrollo Sostenible, 1-7, Bogotá D.C, Colombia (2014)

Rios, L.A. y otros cuatro autores, Producción Alternativa de Biodiésel, Mejoramiento de Propiedades y Valorización de la Glicerina, 1ํe ed., 1-119, Universidad de Antioquia, Medellín, Colombia (2012)

Rodrigues, F.H.A. y otros cinco autores, Comparison between Physico-Chemical Properties of the Technical Cashew Nut Shell Liquid (CNSL) and those Natural Extracted from Solvent and Pressing, Polímeros: 21(2), 156-160 (2011)

Sánchez, E.A. y otros 5 autores, Biodiesel production and de-oiled seed cake nutritional values of a Mexican edible Jatropha curcas. Renewable Energy: 76, 143-147 (2015)

Sarin, A. y otros tres autores, Natural and Synthetic Antioxidants: Influence on the Oxidative Stability of Biodiesel Synthesized from Non - Edible Oil, Energy: 35(12), 4645-4648 (2010)

UNE-EN 14103:2011, Productos derivados de aceites y grasas. Ésteres metílicos de ácidos grasos (FAME). Determinación de los contenidos de éster y de éster metílico del ácido linolénico, 1-16, España (2011)

UNE-EN 14112:2003, Derivados de aceites y grasas. Ésteres metílicos de ácidos grasos (FAME). Determinación de la estabilidad frente a la oxidación (ensayo de oxidación acelerada), 1-20, España (2003)

UNE-EN 14214:2013, Productos petrolíferos líquidos. Ésteres metílicos de ácidos grasos (FAME) para motores diesel y equipos de calefacción. Requisitos y métodos de ensayo, 1-22, España (2013)

Xin, J., Imahara H. y Saka, S., Kinetics on the Oxidation of Biodiesel Stabilized with Antioxidant, Fuel: 88 (2), 282-286 (2009)

Zuleta, E.C., Rios, L.A., y Benjumea, P.N., Oxidative Stability and Cold Flow Behavior of Palm, Sacha-Inchi, Jatropha and Castor Oil Biodiesel Blends, Fuel Processing Technology: 102 (0), 96-101 (2012a)

Zuleta, E.C., Rios, L.A. y Cálderon, J.A., Estudio de la Estabilidad Oxidativa de Biodiésel de Aceite de Palma en Contacto con Materiales Metálicos y Poliméricos Automotrices, Ingeniería y Competitividad: 14 (2), 83-90 (2012b) 